RUNNING HEAD: Attention and heart rate to emotional faces in infants

Enhanced Cardiac and Attentional Responding to Fearful Faces in 7-Month-Old Infants

Mikko J. Peltola, Jukka M. Leppänen, \& Jari K. Hietanen

Department of Psychology, University of Tampere, Finland

Address for correspondence:

Mikko Peltola

Human Information Processing Laboratory, Department of Psychology

FIN-33014 University of Tampere, Finland

E-mail: mikko.peltola@uta.fi

Tel. +358335517967

Fax: +358335517710 


\begin{abstract}
Orienting of attention to emotionally negative stimuli is accompanied by rapid heart rate (HR) deceleration, reflecting enhanced attentional and sensory processing. We studied whether similar emotional modulation of cardiac responding is observed in infants. HR and eye movements were recorded from 7-month-old infants while they observed a fearful or happy face that was flanked after $700 \mathrm{~ms}$ by a peripheral distractor for $2000 \mathrm{~ms}$. An attentional bias for fearful faces was indicated by less frequent and longer-latency saccades toward the distractors during fearful than happy trials. HR deceleration was significantly larger during fearful than happy trials on which infants did not make a distractor-directed saccade. For trials with a distractor-directed saccade, no difference between fearful and happy faces emerged. Thus, the bias to attend preferentially to fearful faces is accompanied by a concomitant increase in the cardiac orienting response.
\end{abstract}




\section{Enhanced Cardiac and Attentional Responding to Fearful Faces in 7-Month-Old Infants}

Prioritizing and allocating capacity-limited attentional resources toward stimuli that are most relevant for well-being and survival require functional interaction between brain systems that provide rapid evaluation of the significance of incoming stimuli and systems that orient the focus of attention (Vuilleumier, 2005). For infants, the advent of self-produced locomotion marks an unprecedented challenge for these systems as the infants are now faced with situations in which they must learn to quickly detect and react to possible dangers in the environment and to utilize adults' facial and vocal emotional expressions in regulating their behavior. Instead, for younger infants, caregivers' emotional signalling carries considerably less referential and regulation-oriented functions (Campos et al., 2000). Therefore, the time when infants learn to move independently may be associated with an increased sensitivity in utilizing the emotional content of social signals in guiding attentional resource allocation.

Indeed, research has shown that by the second half of the first year, infant attention becomes significantly biased toward emotionally potent stimuli such as fearful facial expressions. This bias has been repeatedly evidenced in spontaneous looking time preferences favoring fearful over happy faces (Kotsoni, de Haan, \& Johnson, 2001; Leppänen, Moulson, Vogel-Farley, \& Nelson, 2007; Nelson \& Dolgin, 1985) as well as in larger event-related brain potentials (ERPs) that reflect the allocation of processing resources toward attentiongrabbing stimuli (de Haan, Belsky, Reid, Volein, \& Johnson, 2004; Grossmann et al., 2011; Leppänen et al., 2007; Nelson \& de Haan, 1996). Critically, while such attentional effects are observed in 7-month-old infants, they appear to be absent in 5-month-old infants, suggesting a developmental change in processing fearful expressions during this period (Peltola, Leppänen, Mäki, \& Hietanen, 2009). Recent studies using an emotional modification of the overlap paradigm (Aslin \& Salapatek, 1975) have further refined the influence of fearful faces on infant attention by showing that when infants' attention is first engaged on a foveally 
presented face stimulus and subsequently drawn towards a distractor stimulus presented in the visual periphery, distractor-directed eye movements are significantly inhibited (i.e., less probable and longer in latency) by fearful relative to happy and neutral central stimuli (Leppänen et al., 2010; Peltola, Leppänen, Palokangas, \& Hietanen, 2008; Peltola, Leppänen, Vogel-Farley, Hietanen, \& Nelson, 2009).

While the evidence for an attentional bias for fearful faces by 7 months of age is unequivocal, the mechanisms that underlie it, however, are not thoroughly known. It would be of high interest to determine whether the attentional effects reflect cortically mediated endogenous (i.e., voluntary) attentional control processes or whether more automatic modulatory signals emanating from emotion-related circuits such as the amygdala are at play. In adults, the amygdala is implicated in modulating the influence of emotional stimuli on attention. For example, the amygdala may enhance the neural representations of emotional stimuli by increasing the excitability of neurons in sensory cortices (Bentley, Vuilleumier, Thiel, Driver, \& Dolan, 2003) and thereby biasing attention toward stimuli that receive more intense neural processing, possibly at the expense of suppressing responsivity to competing stimuli (Carlson, Reinke, LaMontagne, \& Habib, in press; Pourtois, Schwartz, Seghier, Lazeyras, \& Vuilleumier, 2006).

Although a direct assessment of the role of the amygdala and other subcortical structures in infants' emotion-processing is hindered by methodological limitations, it is relatively easy to obtain physiological measures of autonomic responding that may be mediated by emotion-related brain systems such as the amygdala. In adults, orienting of attention to external stimuli is accompanied by a rapid deceleration (i.e., slowing) of heart rate (HR; Graham \& Clifton, 1966). In an extensive line of research, Lang, Bradley, and others have shown that the HR deceleration response is amplified by emotionally negative stimuli, such as unpleasant scenes or angry faces (Bradley, Lang, \& Cuthbert, 1993; Bradley, 2009; 
Kolassa \& Miltner, 2006; Lang \& Bradley, 2010). Bradley (2009) pointed out that the HR deceleration in humans appears equivalent to "fear bradycardia" of most mammals, i.e., the rapid slowing of heart rate with accompanied behavioral "freezing" when the animal is confronted with a threatening stimulus. The role of the amygdala in the threat-related orienting response was highlighted by Kapp, Supple, and Whalen (1994) who showed that direct stimulation of the amygdala results in HR deceleration and increased cortical arousal in animals. Thus, the HR deceleration response to threat-related cues may be part of a defenserelated orienting reflex that acts to facilitate perceptual processing and extraction of information about potentially significant stimuli (Bradley, 2009). In the absence of actual threat, e.g., in adult studies with repeated presentations of emotional and neutral pictorial stimuli, the cardiac response habituates after the initial repetitions of the stimuli and no longer differentiates between negative and positive/neutral stimuli (Bradley et al., 1993; Bradley, 2009).

While some evidence exists for adult-like augmented HR deceleration to negative pictures in 7- to 10-year-old children (McManis, Bradley, Berg, Cuthbert, \& Lang, 2001), there is a lack of research on whether adult-like emotional enhancement of the cardiac response could be observed already in infancy. HR measures have been extensively used in studying infants' attention to visual stimuli varying in complexity and meaningfulness (e.g., static achromatic dots vs. dynamic film clips; Courage, Reynolds, \& Richards, 2006). Orienting of attention to new stimuli is reflected in a rapid slowing of $\mathrm{HR}$, followed by a sustained HR deceleration which can last up to tens of seconds if the infant engages in voluntary processing of stimulus content (see Reynolds \& Richards, 2007, for a review). The return of HR to baseline levels is typically associated with the termination of active exploration of stimulus content, even while the infant's eyes may still remain on the stimulus (Richards, 1988). Voluntary attentional engagement and active exploration of stimulus 
content is also implicated by findings of significantly smaller distractibility (i.e., lower percentage of attention shifts to peripheral distractor stimuli) during decelerated HR levels than during other HR-defined phases of attention (Lansink \& Richards, 1997).

Recently, Leppänen et al. (2010) measured changes in HR while 7-month-old infants were presented with the overlap task. Fearful, happy, neutral, or Fourier-transformed faces were first displayed on the center of the screen for $1000 \mathrm{~ms}$ and then flanked by a peripheral checkerboard stimulus for $3000 \mathrm{~ms}$. In addition to replicating the behavioral attentional biases (i.e., slower and less frequent saccades away from fearful as compared to the other faces), HR deceleration during the first 1000 ms post-stimulus was significantly steeper for fearful than happy and neutral faces, indicating an enhanced cardiac orienting response toward fearful expressions. As the number of trials included in the HR analyses was relatively small and the infants made a distractor-directed saccade on a majority of these trials, it was not possible to analyze HR as a function of attentional response type (i.e., whether the infant did or did not make a distractor-directed saccade). Therefore, this initial study left open whether the bias to attend preferentially to fearful faces (i.e., inhibition of distractor-directed saccades) is associated with a corresponding modulation of heart rate by showing a relatively stronger and longer-lasting HR deceleration. It was also left open whether repeated stimulus presentation would eventually habituate the HR modulation by fearful faces and thus confine the effect only to the initial phase of stimulus presentation.

The present study aimed at extending the findings of Leppänen et al. (2010) and refining the dynamics of cardiac modulation by fearful faces in infants. The overlap paradigm was slightly modified to obtain a larger number of trials to enable an analysis of HR as a function of attentional response type (i.e., whether the infant did or did not make a distractordirected saccade). To this end, the trial duration was shortened from $4000 \mathrm{~ms}$ to $2700 \mathrm{~ms}$ and stimulus categories were reduced in order to obtain as many as possible trials for fearful and 
happy expressions. It was hypothesized that the behavioral indices of the attentional bias for fearful faces would be replicated. Thus, saccades away from fearful central stimuli were expected to be slower and less frequent than those from happy central stimuli. As a novel measure, we also analyzed the time it took for the infants to re-engage attention to the central stimuli, i.e., the latency of saccades from the distractor back towards the central stimulus. We hypothesized that this latency would be shorter during fearful face trials, indicating infants' heightened interest in fearful expressions. To investigate whether the behaviorally measured differences in attention could be accounted, in fact, by differential rates of habituation, the probability of saccades away from the central stimuli was also analyzed as a function of phase of the testing session (i.e., early vs. late part of the session). As for the HR measures, it was hypothesized that there is a correspondence between the behavioral markers of increased attention and cardiac responding to fearful faces. Thus, the HR deceleration for fearful face trials with no distractor-directed saccade was expected to be larger than the deceleration for happy faces with no distractor-directed saccade. For trials with a distractor-directed saccade, the HR difference between fearful and happy faces was expected to be less prominent. Finally, HR was also analyzed as function of phase of the testing session to determine whether the attention-related cardiac response shows differential habituation rates for fearful and happy faces.

\section{Methods}

\section{Participants}

The participants were 28 infants ( 7 girls) with a mean age of 216 days $(S D=$ 4.2). All infants were born full term ( $\geq 37$ weeks), had a birth weight of $>2400 \mathrm{~g}$ and no history of visual or neurological abnormalities. For the eye movement analyses, data from 3 infants were discarded due to having less than 3 scorable trials for the analysis of saccadic latency and data from one infant were discarded due to very long saccadic latencies (> $2 S D$ 
from group mean). The eye movement analyses were thus performed with 24 infants. For the HR analyses, some participants were excluded due to technical problems in heart rate recording $(n=7)$ or having less than 3 good trials in some stimulus conditions $(n=5)$, thus retaining 16 infants for the $\mathrm{HR}$ analyses. Approval for the project was obtained from the ethics committee of the Pirkanmaa Hospital District and an informed, written consent was obtained from the parent.

\section{Stimuli and Procedure}

The stimuli were color images of happy and fearful facial expressions of two female models. With an approximately $60-\mathrm{cm}$ viewing distance, the faces measured $15.4^{\circ}$ and $10.8^{\circ}$ of vertical and horizontal visual angle, respectively. Prior to data collection, a group of adults $(n=18)$ rated the facial expressions for happiness and fearfulness on a scale from 1 to 7. The ratings confirmed that the happy $(M=5.9)$ and fearful $(M=6.2)$ facial expressions used were considered good examples of the respective emotions. In addition to fearful and happy faces, five trials with a neutral expression were presented during the first 15 trials. Neutral faces were included because the infants who took part in the present study were a subgroup of infants in an ongoing longitudinal project designed to examine the continuity of emotional development from infancy to childhood and this project investigated differences in eye movements toward distractor stimuli from both emotional and neutral faces. The data for the neutral faces are, however, not reported here because the five neutral trials did not provide sufficient data for the HR analyses we report. Finally, 3 to 4 trials with a monkey face were also presented during the testing session as occasional attention-getters. The data for these stimuli are not reported here, either.

Infants were seated on the parent's lap while stimuli were presented on a 19inch computer monitor that was surrounded by black panels. A hidden video camera recorded the infant's looking behavior and enabled the experimenter to control stimulus presentation. 
Infants' heart rate and eye movements were measured during an overlap task (see Leppänen et al., 2010; Peltola et al., 2009, for illustrations). Each trial was started with the presentation of a fixation image (an animated underwater scene from the movie "Finding Nemo" with colorful content covering the whole area of the image) that measured same size as the face stimuli. Once the infant fixated the stimulus, the experimenter pressed a key to present one of the face stimuli on the center of the screen on a white background. After $700 \mathrm{~ms}$, the face was flanked by a peripheral distractor stimulus $13.6^{\circ}$ equiprobably on the left or right for $2000 \mathrm{~ms}$. The distractors were black-and-white vertically arranged circles or a checkerboard pattern, measuring $15.4^{\circ}$ and $4.3^{\circ}$ vertically and horizontally, respectively. During the task, every infant saw only one model's face. Stimuli were presented in random order with the constraint that the same expression was presented no more than twice in a row and the target on the same side of the screen no more than three times in a row. The trials were presented until the infant became inattentive or fussy, with an average of $51.3(S D=7.9$, range $=29-63)$ trials presented throughout the session.

Analysis of the Behavioral Data

An observer who was blind to the stimulus condition coded the videos by using video editing software with frame-by-frame playback (VirtualDubMod 1.5.10.2). Trials with excessive movement, anticipatory eye movements (eye movements commenced within 160 $\mathrm{ms}$ after distractor onset), and incorrect responses (eye movements away from the face that were not directed towards the distractor) were excluded from the analyses. The mean number of trials retained for the analyses of eye movement responses was $35.1(S D=7.6)$. There was a slight difference in the number of scorable trials between fearful $(M=18.5)$ and happy $(M=$ 16.5) faces, $p<.05$. Controlling this difference statistically did not, however, produce any change in the pattern of results and thus will not be considered further. Three eye movement measures for fearful and happy faces were derived from the data. First, saccade probability 
was calculated as the proportion of trials with a distractor-directed saccade out of the total number of scorable trials (i.e., a sum of trials with distractor-directed saccade as well as nosaccade trials on which the child did not move his/her eyes from the face during the whole trial). Second, saccade latency was calculated as the time from the onset of the distractor to the video frame on which the eyes started to shift towards it. Finally, from trials on which the infant shifted his/her eyes from the distractor back to the face, attentional re-engagement latency was calculated as the time from the onset of the distractor-directed saccade to the video frame on which the eyes started to shift back to the face. To establish the reliability of the data coding procedures, another independent observer who was blind to the stimulus condition coded the data from 6 subjects. The interobserver agreement (Cohen's Kappa) for the classification of responses to different response categories (i.e., distractor-directed saccade present, saccade absent, non-scorable trial) was .89. Pearson correlations of the two observers' judgements of the latency of the saccades were on average .97 (range .84-1). Acquisition and Analysis of the HR Data

Electrocardiogram (ECG) was recorded throughout the presentation of the stimuli with two pre-gelled and self-adhesive electrodes placed on the participant's chest. The ECG was band-pass filtered from 0.05 to $30 \mathrm{~Hz}$, amplified with a gain of 1000 (range +/-2750 $\mu \mathrm{V}$; Accuracy .084 $\mu \mathrm{V} / \mathrm{LSB})$, and stored on a computer disk at the sample rate of $1000 \mathrm{~Hz}$ (Neuroscan/Synamps). Offline, the data were analyzed by using an in-house (Matlab-based) algorithm to identify QRS complexes in the ECG signal, and to measure the time intervals between two successive R-waves (i.e., interbeat intervals or IBI). Lengthening of the IBI corresponds to HR deceleration and shortening to HR acceleration. After computer-based detection of R-peaks, the data were manually corrected for falsely detected and missing peaks. As with the eye movement coding, trials with anticipatory eye movements, incorrect responses, and excessive movement causing distortion in the ECG signal were excluded from 
the analyses. An average of $35.9(S D=7.7)$ good trials were retained in the HR analyses. The average numbers of good trials for the HR analyses per condition were 7.0 (fearful/saccade), 11.1 (fearful/no saccade), 9.9 (happy/saccade), and 7.9 (happy/no saccade). The potential effect of the unequal number of trials between conditions was statistically controlled in the HR data analyses. To be included in the statistical analyses of the HR data, a minimum of 3 good trials were required for each of the four conditions. For a period between $500 \mathrm{~ms}$ prestimulus and $3000 \mathrm{~ms}$ post-stimulus within each trial, the IBIs were quantified and assigned to 500 -ms intervals by weighting each IBI by the proportion of the 500-ms interval occupied by that IBI (see Richards \& Turner, 2001). Finally, IBIs were converted to beats per minute (BPM) and averaged across different trials within each condition included in the HR analyses. Thus, for both happy and fearful trials, average HR change during stimulus presentation was analyzed separately for trials on which the infant did or did not make a saccade toward the distractor. The analyses were performed with HR change scores which were calculated by subtracting the BPMs of each post-stimulus 500-ms interval from the BPM during the prestimulus period. Accordingly, negative change score values indicate HR deceleration while positive values indicate HR acceleration during stimulus viewing.

\section{Statistical Analyses}

For the behavioral data, differences between fearful and happy faces in the three eye movement measures were tested with $t$-tests. For the heart rate data, the HR change scores were subjected to a $2 \times 2 \times 6$ repeated measures analysis of variance (ANOVA) with Emotion (fearful, happy), Response (saccade, no saccade), and Time (0-500, 500-1000, 1000-1500, 1500-2000, 2000-2500, 2500-3000 ms) as within-subject factors.

In addition to these main analyses, analyses were also run to examine whether a) differences in the numbers of good trials for fearful and happy faces with and without saccades had an influence on the HR data by entering the difference in the number of trials as 
a covariate in the ANOVA, and b) whether the behavioral and HR results reflect differential rates of habituation between fearful and happy faces. For the habituation analyses, the behavioral and HR data were split in half and Phase (i.e., the early vs. late parts of the stimulus presentation session) was included as a factor in the ANOVA. For the HR data, the Emotion $\times$ Phase $\times$ Time interaction (i.e., collapsed across the two response types) was analyzed with the same number of subjects as in the main HR analyses. To analyze the influence of Phase separately for saccade and no saccade trials in the HR data, the exclusion criterion was made a bit less stringent to avoid the exclusion of an excessive number of infants from these analyses. Thus, each infant had to provide a minimum of 2 good saccade or no saccade trials during both the early and late phases of the session to be included in the habituation analyses. Ten infants fulfilled this criterion for the saccade condition and 12 infants for the no saccade condition.

In all analyses, the degrees of freedom were corrected for sphericity assumption violations by using the Greenhouse-Geisser correction procedure. Bonferroni correction was applied for the $p$-values in cases where the number of pairwise comparisons was higher than three.

Results

\section{Behavioral Measures}

The probability of making a distractor-directed saccade was significantly lower during trials with fearful $(M=.47)$ than happy $(M=.60)$ central stimulus face, $t(23)=4.2, p<$ .001 (Figure 1). Emotional expression of the central stimulus exerted significant effects also on eye movement latencies. Thus, the infants were slower to initiate a saccade away from fearful (749 ms) than happy faces $(670 \mathrm{~ms}), t(23)=2.3, p<.05$ and when they re-engaged their attention on the central stimulus, the eyes were shifted more quickly toward a fearful (587 ms) than a happy face $(663 \mathrm{~ms}), t(22)=2.4, p<.05$. 
Figure 1 about here

\section{Heart Rate Measures}

The $2 \times 2 \times 6$ ANOVA revealed a main effect of Emotion, $F(1,15)=7.9, p<$ $.05, \eta_{\mathrm{p}}{ }^{2}=.34$ as well as an Emotion $\times$ Time interaction, $F(5,75)=7.5, p \leq .001, \varepsilon=.55, \eta_{\mathrm{p}}{ }^{2}=$ .33. Pairwise comparisons (with Bonferroni correction) indicated that the significantly larger HR deceleration for fearful faces emerged by $1500 \mathrm{~ms}$ after stimulus onset, $t(15)=3.0, p<$ .05 and that it remained significant also during the 2000-ms, $t(15)=3.5, p<.05$, and 2500-ms time windows, $t(15)=4.0, p<.01$. A significant Emotion $\times$ Response interaction was also observed, $F(1,15)=5.0, p<.05, \eta_{\mathrm{p}}{ }^{2}=.25$ (see Figure 2$)$. Therefore, Emotion $(2) \times$ Time $(6)$ ANOVAs were conducted separately for saccade and no saccade trials. For trials with a distractor-directed saccade, the main effect of Time approached significance, $F(5,75)=2.8, p$ $<.09, \varepsilon=.35, \eta_{\mathrm{p}}{ }^{2}=.16$ but there was no effect of facial expression on HR change, $F(1,15)=$ $.2, p>.6, \eta_{\mathrm{p}}{ }^{2}=.02$ or an Emotion $\times$ Time interaction, $F(5,75)=1.6, p>.2, \varepsilon=.52, \eta_{\mathrm{p}}{ }^{2}=.10$. Significant differences emerged, however, during the no saccade trials, resulting in main effects for Emotion, $F(1,15)=19.7, p<.001, \eta_{\mathrm{p}}{ }^{2}=.57$, Time, $F(5,75)=3.4, p<.05, \varepsilon=.43$, $\eta_{\mathrm{p}}{ }^{2}=.18$, and an Emotion $\times$ Time interaction, $F(5,75)=8.6, p \leq .001, \varepsilon=.39, \eta_{\mathrm{p}}{ }^{2}=37$. These effects were due to larger HR deceleration during fearful than happy face trials, with significant differences starting by $1000 \mathrm{~ms}$ after stimulus onset, $t(15)=3.0, p<.06$, and sustaining through the 1500-ms, $t(15)=4.3, p<.01,2000-\mathrm{ms}, t(15)=4.6, p<.01$, and 2500ms time windows, $t(15)=4.8, p<.01$.

Figure 2 about here 


\section{Additional Analyses}

As the infants had a lower probability of making a distractor-directed saccade during fearful trials, there was also a significant difference in the number of good trials accepted for the HR analyses between fearful faces with no saccade $(M=11.1)$ and happy faces with no saccade $(M=7.9), t(15)=3.5, p<.01$. To control for the possible influence of this difference on the observed response-related differences in HR deceleration, the HR data for trials with no saccade were re-analyzed with the difference in the number of trials (i.e., fearful trials with no saccade - happy trials with no saccade) entered as a covariate in the ANOVA. As a result, the main effect of Emotion remained significant, $F(1,14)=11.6, p<$ $.01, \eta_{\mathrm{p}}{ }^{2}=.45$, indicating no significant effect produced by the difference in the number of trials on the observed pattern of results.

To measure habituation, the behavioral data were subjected to a $2 \times 2$ ANOVA with Emotion (fearful, happy) and Phase (early, late) as within-subject factors. There were main effects for Emotion, $F(1,23)=19.5, p<.001, \eta_{\mathrm{p}}^{2}=.46$, and Phase, $F(1,23)=8.0, p \leq$ $.01, \eta_{\mathrm{p}}{ }^{2}=.26$, but no interaction between them, $F(1,23)=.2, p>.6, \eta_{\mathrm{p}}{ }^{2}=.01$. These effects resulted from the probability of making a distractor-directed saccade being smaller during the second half of the experiment but this habituation was similar for fearful and happy face trials. For HR, collapsing the data across the two eye movement response types, an Emotion $(2) \times$ Phase $(2) \times$ Time (6) ANOVA replicated the main effect of Emotion, $F(1,15)=9.0, p<$ $.01, \eta_{\mathrm{p}}{ }^{2}=.37$, and an Emotion $\times$ Time interaction, $F(5,75)=7.4, p \leq .001, \varepsilon=.52, \eta_{\mathrm{p}}{ }^{2}=.33$. However, no main effect of Phase or any interactions involving Phase were observed, $p s>.3$. Thus, the differences in HR deceleration between fearful and happy faces remained constant throughout the experiment. The separate analyses for the HR data on saccade and no saccade trials showed effects identical to the main analyses reported above and, critically, Phase did not interact with Emotion or with the Emotion $\times$ Time interaction on the no saccade trials, $p \mathrm{~s}$ 
> .6. In other words, the stronger HR deceleration during fearful face trials with no distractordirected saccades persisted throughout the experiment.

Finally, as the main analyses indicated that the significant differences in cardiac responding between fearful and happy faces occurred at later time windows than in Leppänen et al. (2010), we wanted to test whether the early-occurring cardiac modulation that was observed by Leppänen et al. (i.e., within the first $1000 \mathrm{~ms}$ ) could nevertheless be replicated when the conditions of the analysis are matched as closely as possible between these two studies. Thus, we analyzed HR changes within the first two time windows after stimulus onset (i.e., 0-1000 ms) during the early phase of the testing session which resembles the study of Leppänen et al. in terms of the number of trials included in the analysis and the time required to accumulate these trials. A 2 (Emotion) $\times 2$ (Time) ANOVA mirrored the results of Leppänen et al. (2010) in that a significant Emotion $\times$ Time interaction was observed, $F(1$, $15)=4.4, p=.052, \eta_{\mathrm{p}}^{2}=.23$. This was due to fearful faces showing a steeper cardiac deceleration within the first $1000 \mathrm{~ms}$ of the trial during the first half of the testing session. A similar interaction was absent during the later phase of the testing session, $p>.4$.

\section{Discussion}

This study aimed at elucidating the dynamics of cardiac responding during changes in infants' attention between emotional faces and non-emotional distractor stimuli. The behavioral markers of selective attention replicated previous findings (Leppänen et al., 2010; Peltola et al., 2008; Peltola, Leppänen, Vogel-Farley et al., 2009) by showing that saccades toward the distractor stimuli were less frequent and of longer latency from fearful than happy central stimuli. As a novel finding, we also observed that when the infants shifted their attention back to the still-visible central stimulus, this occurred significantly faster to fearful than happy central stimuli. The analyses of heart rate change during stimulus viewing showed that fearful central stimuli were accompanied with a larger cardiac deceleration response. This difference 
appears to be driven by pronounced HR deceleration during fearful trials on which the infant did not make a distractor-directed saccade.

These data are consistent with the view that by the second half of the first year, infants acquire an attentional bias toward fearful emotional expressions (Leppänen \& Nelson, 2009; Peltola, Leppänen, Mäki et al., 2009). The reasons for the heightened interest in fearful faces are not perfectly clear. Although it would be tempting to suggest that the emotional signal value of fearful faces is driving the attentional effects, the available data are not yet entirely sufficient to confirm that infants would actually be able to derive and respond to the emotional meaning of fearful expressions. Alternatively, it has been proposed (Nelson \& de Haan, 1996; Vaish, Grossmann, \& Woodward, 2008) that the attentional bias might simply reflect a novelty preference, i.e., the fact that fear is expressed by the caregivers considerably less often than happiness in an infant's typical rearing environment (Malatesta \& Haviland, 1982) and infants are known to pay more attention to novel stimuli (Fantz, 1964). A noveltybased account is challenged, however, by studies showing that for 6-month-olds, images of the mother's face (which is, of course, a familiar but an emotionally meaningful stimulus) elicit stronger attention-sensitive ERPs than an unfamiliar female's face (de Haan \& Nelson, 1997, 1999) and posed expressions that are rated as novel and non-emotional do not produce similar effects as fearful faces on saccadic probability in the overlap task (Peltola et al., 2008). Moreover, the findings by Hoehl and colleagues showing context-dependent modulation of attention-sensitive ERPs in 7-month-old infants by the gaze direction of angry (Hoehl \& Striano, 2008) and fearful (Hoehl, Palumbo, Heinisch, \& Striano, 2008) faces indicate a level of sophistication in infants' emotional face processing that is not easily interpreted as an attentional preference for more novel stimuli. Finally, the absence of an attentional bias for fearful faces in 5-month-old infants (Peltola, Leppänen, Mäki et al., 2009), for whom fearful 
faces are undoubtedly as novel as for 7-month-olds, also argues against a novelty-based account.

In addition to the emotional modulation of saccades away from the central stimulus, fearful faces were also associated with a faster re-engagement of attentional focus on them. Whether or not this effect reflects a response to the emotional signal value of a fearful expression, it nevertheless indicates that infants actively control their attention to efficiently extract information from fearful faces. They devote a longer time to process fearful expressions and terminate the scanning of other information in the visual field more quickly in order to continue processing the fearful expression. The persistent bias to prefer fearful expressions over other stimuli presumably enables relatively efficient associative learning in the contexts in which fearful emotions are expressed by the caregivers (e.g., situations involving impending danger).

Increased allocation of attentional resources is also proposed by the HR data which showed a larger HR deceleration to fearful faces, replicating the findings of Leppänen et al. (2010). Both data are in line with previous adult studies showing augmented HR deceleration to emotionally negative visual stimuli that arises during the first seconds of stimulus viewing (Bradley et al., 1993; Kolassa \& Miltner, 2006). The present study aimed at extending the finding of a generally larger HR deceleration for fearful faces with a modification of the overlap paradigm that enabled segregating the HR data into different attentional response categories and made it possible to relate cardiac responding more closely with the level of attentional engagement to fearful and happy faces.

Indeed, analyses of HR change during different eye movement responses on fearful and happy face trials revealed that the bias to attend preferentially to fearful faces (i.e., lower frequency of saccades away from fearful faces) is associated with a concomitant increase in the cardiac orienting response (i.e., larger HR deceleration during fearful than 
happy face trials with no distractor-directed saccade). The cardiac response was robust as it did not markedly habituate during repeated presentations of the stimuli. These findings strongly suggest that the infant's response of retaining attention on a fearful face regardless of the presence of distractor stimuli is due to a persistent capture of attention by a fearful face and not, for example, disinterest or fatigue that would lead to inattentiveness to peripheral stimulation while the testing session proceeds. It is also noteworthy that the cardiac responses on happy trials with no distractor-directed saccades did not show a clear HR deceleration early or late during the experiment. Thus, it was not the case that the inhibition of attention shifts during happy face trials would have been associated with a cardiac deceleration during the initial presentations of happy central stimuli but subsequently dissipated. The difference between fearful and happy faces was also not due to fearful faces with no distractor-directed saccades having more trials included in the analysis. Whether the saccadic inhibition to fearful as compared to happy faces represents a difference in attentional processing that would be best described as quantitative (i.e., larger allocation of resources) or qualitative (i.e., implying different or additional mediating mechanisms) is not directly revealed by the present data.

A crucial advancement in uncovering the mechanism that underlie the augmented cardiac responding during the perception of fearful faces would be to determine whether the attentional effects result from cortically mediated endogenous (i.e., voluntary) attentional control processes or whether the attentional bias toward fear reflects a more obligatory response that may be partly mediated by emotion-related circuits such as the amygdala. According to Richards' model of heart rate and attention (e.g., Richards, 2008), the HR deceleration during increased attentional engagement on a stimulus is related to a nonspecific arousal mechanism that acts to "energize" cortical processing of the attended stimulus. The effects of such arousal on the heart are thought to operate through the reticular 
formation (Mesulam, 1983) which receives signals from the cardioinhibitory centers in the frontal cortex and further connects to parasympathetic fibers to slow down heart rate (Richards, 2008). The sustained HR deceleration which is associated with active and voluntary attentional engagement to the stimulus (Hicks \& Richards, 1998; Lansink \& Richards, 1997) is considered to result from a continuing activation of the arousal network (Richards, 2008).

The present results could be seen as discordant with the Richards' model as the happy face trials with no distractor-directed saccades (i.e., an overt indication of increased attention to the central stimulus) did not show a clear HR deceleration. However, because of the short trial duration and rapid rhythm of stimulus changes in the present study, it may not be reasonable to directly interpret the present data in light of the Richards' model which is primarily based on results obtained from continuous and longer-lasting visual stimulation or object exploration (e.g., Lansink \& Richards, 1997). While the absence of a clear HR deceleration for happy faces apparently contradicts with the behaviorally observed inhibition of responding to the distractor stimuli, the data are nevertheless in line with the hypothesis that the degree of HR deceleration generally corresponds to the depth of encoding the stimulus at fixation (Blaga \& Colombo, 2006; Reynolds \& Richards, 2007). Thus, happy faces, whether attended throughout the trial or not, may be encoded in a shallower manner which is reflected in less prominent changes in HR. The difference between fearful and happy faces would thus reflect a quantitative difference in the attentional response. Finally, whether infants' attentional engagement to fearful faces could be shown to be associated with a sustained HR deceleration (i.e., more directly indicating endogenous control of attention) presumably requires presenting the stimuli for a longer period. This would help in revealing variation in attentional state after the initial orienting to the stimulus has occurred. 
Another interpretation of the data with less emphasis on a cortical influence would posit that the cardiac deceleration is an obligatory, reflex-like response to stimuli associated with threat or novelty that is modulated by an influence of the amygdala on the brainstem structures controlling parasympathetic innervation to the heart. According to Lang and Bradley (Bradley, 2009; Lang \& Bradley, 2010), the cardiac response to threat-related stimuli is part of a cascade of defensive reflexes which also involve changes in other autonomic functions (e.g., respiration and electrodermal activity). The purpose of these defense-related reactions is to serve the enhanced gathering of information about the potentially significant stimulus and to prompt the individual for adaptive behavioral responding (i.e., withdrawal or attack in the face of an actual threat). According to this framework, the HR deceleration to fearful faces in the present study may thus reflect a relatively automatic and subcortically mediated autonomic adjustment that serves to increase information-gathering from fearful faces and thereby contribute to the stronger suppression of distractor-directed saccades during fearful than happy face trials. Such a mechanism could also more readily account for the dissociation of HR changes from actual attentional performance during happy face trials. Thus, while infants do pay attention to happy faces and inhibit attention shifts toward the distractors, such responses may not be associated with an automatic modulation of autonomic responses, biasing the infants toward increased visual processing during the perception of fearful faces.

In conclusion, the present results showed behavioral attentional biases and adultlike autonomic responding to threat-related stimuli and corroborate the view that by 7 months of age, infants show rapid and persistent prioritization of fearful expressions over happy faces. Whether the cardiac effects specifically emerge during this age period when infants typically begin to move independently (and when adults' emotional expressions also start to gain more referential and regulative purposes) or whether the effects are observed also in 
younger infants would be a highly interesting area of exploration. Future studies should also explicate the potential mechanisms conveying the effects of fearful faces on infants' attention in further detail by measuring brain activity while infants are presented with emotional and competing stimuli as well as by examining HR changes during emotional face perception over longer time scales in order to parse the effects of emotion on the different HR-defined phases of attention. 


\section{References}

Aslin, R. N., \& Salapatek, P. (1975). Saccadic localization of visual targets by the very young human infant. Perception and Psychophysics, 17, 293-302.

Bentley, P., Vuilleumier, P., Thiel, C. M., Driver, J., \& Dolan, R. J. (2003). Cholinergic enhancement modulates neural correlates of selective attention and emotional processing. Neuroimage, 20, 58-70.

Blaga, O. M., \& Colombo, J. (2006). Visual processing and infant ocular latencies in the overlap paradigm. Developmental Psychology, 42, 1069-1076.

Bradley, M. M. (2009). Natural selective attention: Orienting and emotion. Psychophysiology, $46,1-11$.

Bradley, M. M., Lang, P. J., \& Cuthbert, B. N. (1993). Emotion, novelty, and the startle reflex: Habituation in humans. Behavioral Neuroscience, 107, 970-980.

Campos, J. J., Anderson, D. I., Barbu-Roth, M. A., Hubbard, E. M., Hertenstein, M. J., \& Witherington, D. (2000). Travel broadens the mind. Infancy, 1, 149-219.

Carlson, J. M., Reinke, K. S., LaMontagne, P. J., \& Habib, R. (in press). Backward masked fearful faces enhance contralateral occipital cortical activity for visual targets within the spotlight of attention. Social Cognitive and Affective Neuroscience.

Courage, M. L., Reynolds, G. D., \& Richards, J. E. (2006). Infants' attention to patterned stimuli: Developmental change from 3 to 12 months of age. Child Development, 77, 680695. 
de Haan, M., Belsky, J., Reid, V., Volein, A., \& Johnson, M. H. (2004). Maternal personality and infants' neural and visual responsivity to facial expressions of emotion. Journal of Child Psychology and Psychiatry, 45, 1209-1218.

de Haan, M., \& Nelson, C. A. (1997). Recognition of the mother's face by six-month-old infants: A neurobehavioral study. Child Development, 68, 187-210.

de Haan, M., \& Nelson, C. A. (1999). Brain activity differentiates face and object processing in 6-month-old infants. Developmental Psychology, 35, 1113-1121.

Fantz, R. L. (1964). Visual experience in infants: Decreased attention to familiar patterns relative to novel ones. Science, 146, 668-670.

Graham, F. K., \& Clifton, R. K. (1966). Heart-rate change as a component of the orienting response. Psychological Bulletin, 65, 305-320.

Grossmann, T., Johnson, M. H., Vaish, A., Hughes, D., Quinque, D., Stoneking, M., et al. (2011). Genetic and neural dissociation of individual responses to emotional expressions in human infants. Developmental Cognitive Neuroscience, 1, 57-66.

Hicks, J. M., \& Richards, J. E. (1998). The effects of stimulus movement and attention on peripheral stimulus localization by 8- to 26-week-old infants. Infant Behavior and Development, 21, 571-589.

Hoehl, S., Palumbo, L., Heinisch, C., \& Striano, T. (2008). Infants' attention is biased by emotional expressions and eye gaze direction. Neuroreport, 19, 579-582.

Hoehl, S., \& Striano, T. (2008). Neural processing of eye gaze and threat-related emotional facial expressions in infancy. Child Development, 79, 1752-1760. 
Kapp, B. S., Supple, W. F.,Jr, \& Whalen, P. J. (1994). Effects of electrical stimulation of the amygdaloid central nucleus on neocortical arousal in the rabbit. Behavioral Neuroscience, 108, 81-93.

Kolassa, I. T., \& Miltner, W. H. (2006). Psychophysiological correlates of face processing in social phobia. Brain Research, 1118, 130-141.

Kotsoni, E., de Haan, M., \& Johnson, M. H. (2001). Categorical perception of facial expressions by 7-month-old infants. Perception, 30, 1115-1125.

Lang, P. J., \& Bradley, M. M. (2010). Emotion and the motivational brain. Biological Psychology, 84, 437-450.

Lansink, J. M., \& Richards, J. E. (1997). Heart rate and behavioral measures of attention in six-, nine-, and twelve-month-old infants during object exploration. Child Development, $68,610-620$

Leppänen, J., Peltola, M. J., Mäntymaa, M., Koivuluoma, M., Salminen, A., \& Puura, K. (2010). Cardiac and behavioral evidence for emotional influences on attention in 7 month-old infants. International Journal of Behavioral Development, 34, 547-553.

Leppänen, J. M., Moulson, M. C., Vogel-Farley, V. K., \& Nelson, C. A. (2007). An ERP study of emotional face processing in the adult and infant brain. Child Development, 78, 232-245.

Leppänen, J. M., \& Nelson, C. A. (2009). Tuning the developing brain to social signals of emotions. Nature Reviews Neuroscience, 10, 37-47. 
Malatesta, C. Z., \& Haviland, J. M. (1982). Learning display rules: The socialization of emotion expression in infancy. Child Development, 53, 991-1003.

McManis, M. H., Bradley, M. M., Berg, W. K., Cuthbert, B. N., \& Lang, P. J. (2001). Emotional reactions in children: Verbal, physiological, and behavioral responses to affective pictures. Psychophysiology, 38, 222-231.

Mesulam, M. M. (1983). The functional anatomy and hemispheric specialization for directed attention: The role of the parietal lobe and its connectivity. Trends in Neurosciences, 6 , 384-387.

Nelson, C. A., \& de Haan, M. (1996). Neural correlates of infants' visual responsiveness to facial expressions of emotion. Developmental Psychobiology, 29, 577-595.

Nelson, C. A., \& Dolgin, K. G. (1985). The generalized discrimination of facial expressions by seven-month-old infants. Child Development, 56, 58-61.

Peltola, M. J., Leppänen, J. M., Mäki, S., \& Hietanen, J. K. (2009). Emergence of enhanced attention to fearful faces between 5 and 7 months of age. Social Cognitive and Affective Neuroscience, 4, 134-142.

Peltola, M. J., Leppänen, J. M., Palokangas, T., \& Hietanen, J. K. (2008). Fearful faces modulate looking duration and attention disengagement in 7-month-old infants. Developmental Science, 11, 60-68.

Peltola, M. J., Leppänen, J. M., Vogel-Farley, V. K., Hietanen, J. K., \& Nelson, C. A. (2009). Fearful faces but not fearful eyes alone delay attention disengagement in 7-month-old infants. Emotion, 9, 560-565. 
Pourtois, G., Schwartz, S., Seghier, M. L., Lazeyras, F., \& Vuilleumier, P. (2006). Neural systems for orienting attention to the location of threat signals: An event-related fMRI study. Neuroimage, 31, 920-933.

Reynolds, G. D., \& Richards, J. E. (2007). Infant heart rate: A developmental psychophysiological perspective. In L. A. Schmidt, \& S. J. Segalowitz (Eds.), Developmental psychophysiology: Theory, systems, and methods (pp. 173-212). Cambridge, UK: Cambridge University Press.

Richards, J. E. (1988). Heart rate offset responses to visual stimuli in infants from 14 to 26 weeks of age. Psychophysiology, 25, 278-291.

Richards, J. E. (2008). Attention in young infants: A developmental psychophysiological perspective. In C. A. Nelson, \& M. Luciana (Eds.), Handbook of developmental cognitive neuroscience (2nd ed., pp. 479-497). Cambridge, MA: MIT Press.

Richards, J. E., \& Turner, E. D. (2001). Extended visual fixation and distractibility in children from six to twenty-four months of age. Child Development, 72, 963-972.

Vaish, A., Grossmann, T., \& Woodward, A. (2008). Not all emotions are created equal: The negativity bias in social-emotional development. Psychological Bulletin, 134, 383-403.

Vuilleumier, P. (2005). How brains beware: Neural mechanisms of emotional attention. Trends in Cognitive Sciences, 9, 585-594. 
Author notes

This work was supported by the Finnish National Doctoral Programme of Psychology and the Academy of Finland (projects \#131786, 130272, 130895, and 218284). 
Figure Captions

Figure 1. The eye movement data for fearful and happy faces displaying average saccade probability (top), the latency of making a distractor-directed saccade (middle), and the latency of making a saccade back towards the face (bottom).

Figure 2. Average HR change during fearful and happy face trials as a function of attentional response type. 

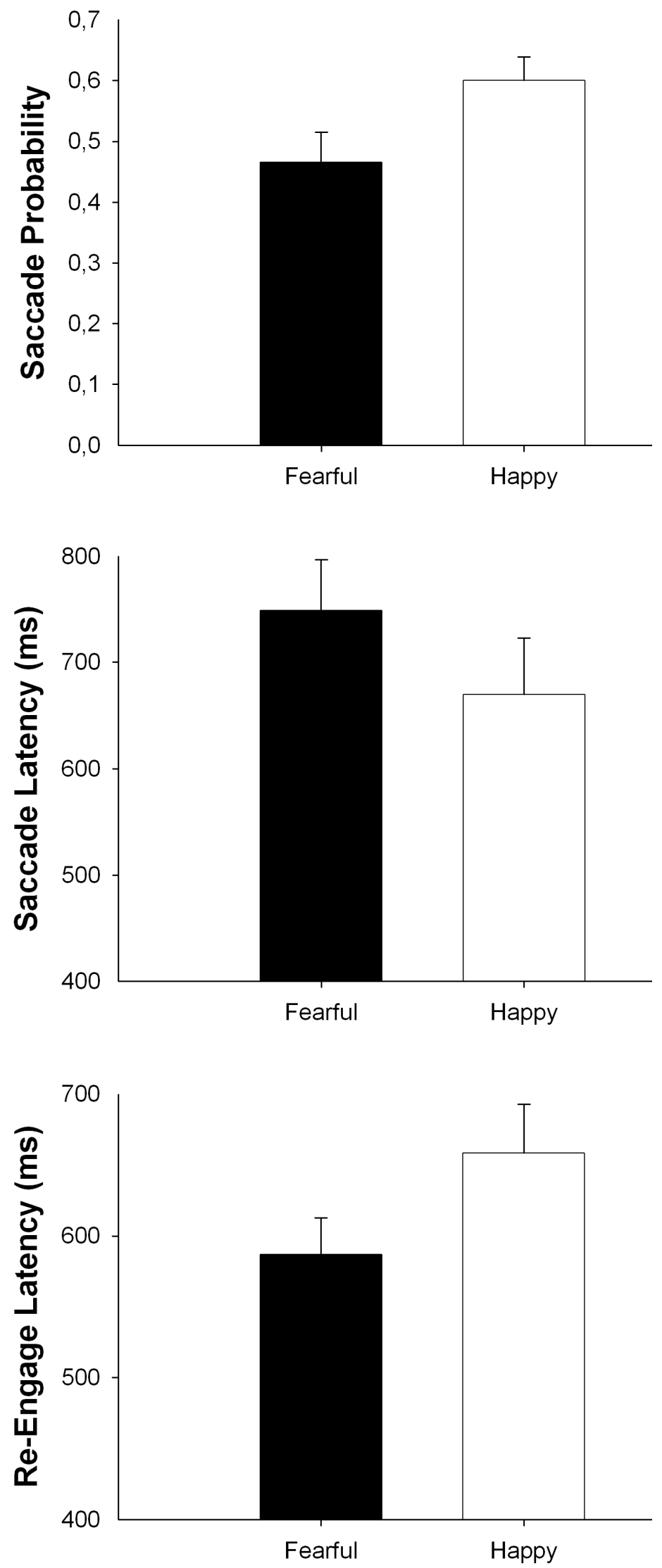

Figure 1. 


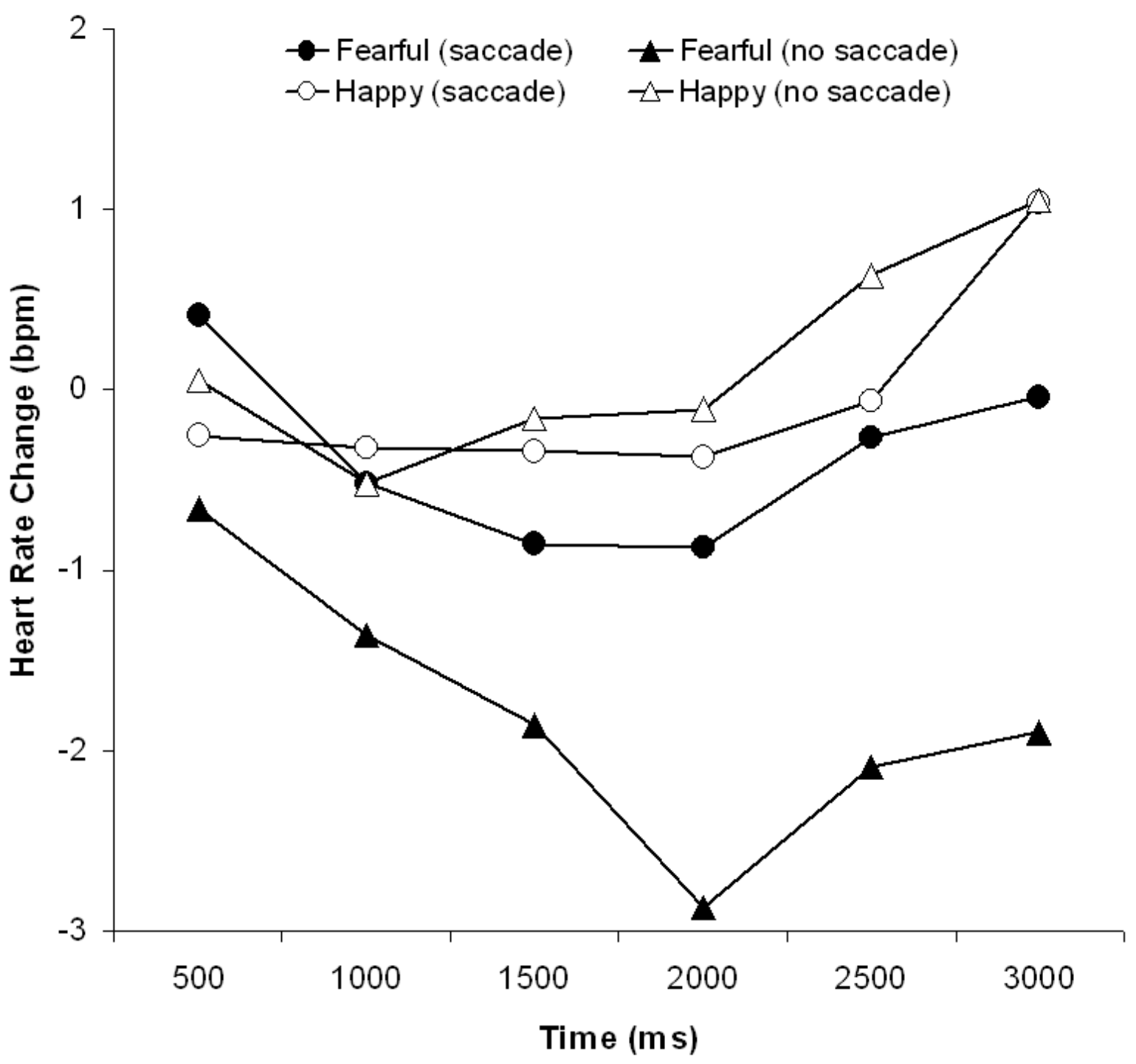

Figure 2. 